\title{
Analysis of a Thermal Plasma Diamond GVD System
}

D. Kolman

This paper deals with the analysis of a typical engineering system utilizing thermal plasma - a system for Diamond Chemical Vapor Deposition. It defines the system - a slightly overexpanded plasma jet impinging at a downstream-located substrate, outlines the theoretical description of the system - the Navier-Stokes and species conservation equations, and presents key theoretical results on the major and most troublesome factors influencing diamond deposition - velocity and temperature of the jet. Then, the paper demostrates the necessity to shift from a laminar to a turbulent flow description and compares both results to experiments. An explanation of the remaining discrepancy insufficient velocity drop in the jet - is attempted.

Keywords: modeling, thermal plasma, CVD.

\section{Introduction}

Thermal plasmas are plasmas in which the thermodynamic state approaches Local Thermodynamic Equilibrium (LTE). Although such plasmas are characterized by a single temperature common to all species and to all their thermal movements (translation, rotation, vibration, electronic temperature), the analysis of a thermal plasma system is highly complex. It involves an intricate interplay between fluid dynamics, turbulent transport, thermal radiation, chemical reactions and interaction with other phases.

This paper deals with the theoretical and experimental analysis of a typical engineering system utilizing thermal plasma - a system for Chemical Vapor Deposition (CVD), in particular deposition of diamond. The system under con-

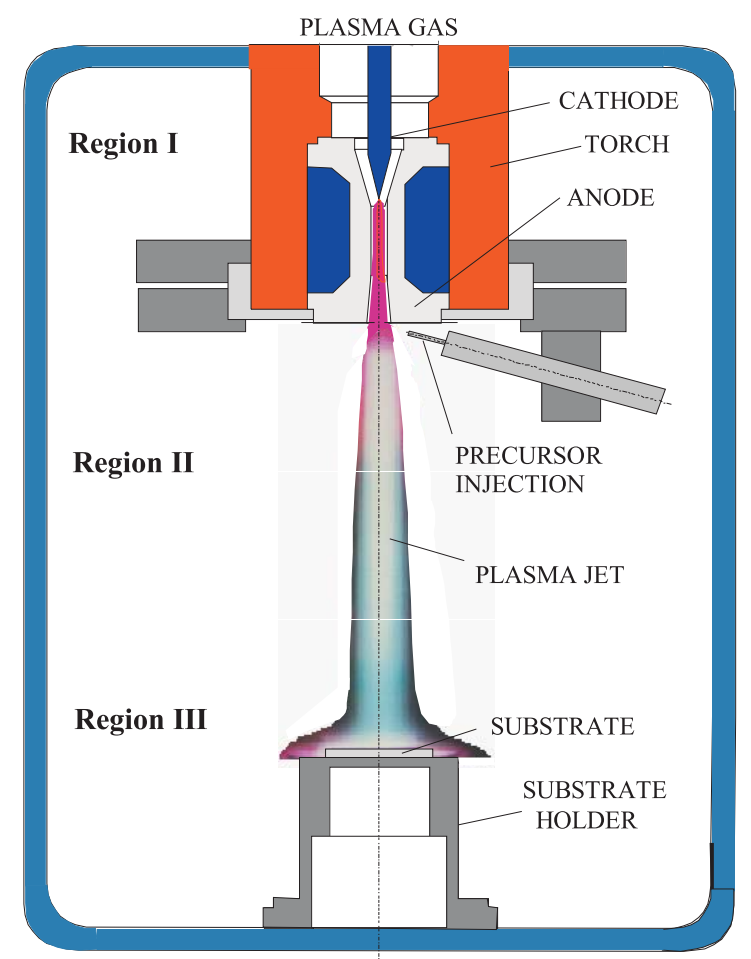

Fig. 1: The thermal plasma diamond CVD system sideration is shown schematically in Figure 1, where the major processes are: (1) heating of the process gas with a DC or an RF plasma torch, (2) expansion of the gas into the reactor and introduction of the diamond growth precursor species, and (3) interaction of the jet with the substrate, and diamond formation. The focus of this paper is on the second stage - the interaction of the plasma flow (plasma jet) with the surrounding atmosphere and with the substrate located downstream.

The DC torch power of the system under consideration is $6.3 \mathrm{~kW}$, the jet power is about $50 \%$ of this, i.e. $3.1 \mathrm{~kW}$. The argon flow rate through the torch is 16 SLM, and the hydrogen flow rate through the torch is 5 SLM. Methane is used as a diamond growth precursor and is injected through three probes within $3 \mathrm{~mm}$ of the torch nozzle exit with a total flow rate of $0.25 \mathrm{SLM}$. The reactor pressure is $12.5 \mathrm{kPa}$ the substrate stand-off distance is $8 \mathrm{~cm}$. The plasma torch exit temperature is of the order of $5000 \mathrm{~K}$ and the torch exit velocity is of the order of $2500 \mathrm{~m} / \mathrm{s}$. The system is located and operates at FG Plasma-Oberflächentechnik, Technische Universität Ilmenau, Thüringen, Germany.

\section{Laminar flow modeling}

In this study, it was assumed that the gas exits the plasma torch with known radial profiles of pressure, velocity, enthalpy and chemical composition. These boundary conditions were obtained in two ways: (1) by a simple axial integration of the conservation equations with a $1 \mathrm{D}$ model of the torch region, and (2) from the spectroscopic measurements made by Jahn [1] on the plasma system under consideration. Jahn measured the $\mathrm{H}_{\beta}$ lines in the region close to the torch nozzle exit and from the results evaluated the plasma jet temperature. The temperature was evaluated assuming an LTE in the torch exit. In reality, the specification of the inflow boundary conditions turns out to be not so much an input but rather one of the outputs of the design analysis, and the selected final profiles are based on the best agreement of the overall simulation results with experiments.

Assuming the upstream boundary conditions are known, one forms a set of conservation equations that are to be solved for the unknown flow characteristics, temperature/energy, pressure and species concentrations. The set includes [2] the 
continuity equation, two momentum equations, the energy equation, species continuity equations with temperature-dependent chemical kinetics, and as a closure the equation of the state. The species set may but does not include ions due to a rather low jet temperature in the region of interest. None of the viscous and/or diffusion effects, and compressibility effects can be excluded. As there is no electric arc passing through the reactor domain there is no need to solve the Maxwell-Boltzmann electromagnetic equations. Thermal diffusion terms and radiation are also neglected due to their small values in the situation under consideration (a rather low temperature).

The obtained equation set is solved with a finite difference semi-implicit pressure-based algorithm for compressible viscous flows with an arbitrary number of conservation equations [3]. The final form of the program adopts a hybrid convection-diffusion flux approximation at the computational cell interfaces. The 2D computational domain includes the reactor and the substrate and is non-uniformly subdivided into $\sim 100$ computational cells in both directions with the finest spacing in the vicinity of the precursor injection location and at the substrate.

At the substrate, no slip boundary conditions are adopted for the momentum equation, the known radial temperature distribution is imposed at the substrate surface, and a source/sink is included in the species conservation equation where the strength of the surface net production rates is given by a system of surface chemical reactions. For this purpose the momentary chemical state of the surface is described via concentrations of appropriately defined surface species (a radical site, a site ended with a hydrogen atom, etc.). Energy release by surface recombination is included in the energy balance.

In the present model, the $\mathrm{Ar}-\mathrm{H}_{2}-\mathrm{CH}_{4}$ kinetics mechanism consists of 34 gas phase species $\left(\mathrm{Ar}, \mathrm{H}, \mathrm{H}_{2}, \mathrm{CH}_{0-4} \mathrm{C}_{2} \mathrm{H}_{0-6}\right)$ and 52 gas phase reactions. The production rates of the individual species are given by the sum of contributions from the individual gas phase reactions. All temperature and chemical composition-dependent thermodynamic and transport properties are evaluated using the Wilke mixing rule which has been found to be sufficient for the situation under consideration. Equilibrium chemical composition is assumed in the torch exit. For a discussion of the surface chemistry see [2]. In all the figures presented, the jet is assumed slightly overexpanded with the nozzle pressure $\sim 9000 \mathrm{~Pa}$, axial velocity $2700 \mathrm{~m} / \mathrm{s}$ and temperature $\sim 4150 \mathrm{~K}$. (The supersonic character of the jet, $\mathrm{M} \sim 2$, is supported by the diamond shocks sometimes visible in the actual jet.)

After the theoretical model was developed and the results obtained, a set of enthalpy probe measurements [4] was performed in the reactor - see Figure 2. These data were used to validate the theoretical results, through a comparison to gain more advanced insight into the deposition process, and to suggest the further course of investigation. In evaluation of the measurements it was assumed that the static pressure of the jet is close to the chamber pressure - a reasonable assumption far away from the torch exit (an assumption of this kind is necessary to evaluate the results across the bow shock formed in front of the enthalpy probe in the case of compressible high velocity flows).
To the disappointment of the author it was found that the experimental data agree rather poorly with the predictions. The main problem is that both the axial velocity and the jet enthalpy are too high and do not fall off as fast as the experiment suggests. After a detailed analysis with the experimental researchers the following explanations have been proposed:

(1) The spectroscopic data are too high: when computing the temperature from the $\mathrm{H}_{\beta}$ line data a state of LTE was assumed. However, this is not likely to be the case in the torch exit, where electron temperature tends to be elevated above the heavy species temperature. Thus the temperature derived from the spectroscopic measurements corresponds more to the electron temperature than to the heavy species temperature and should not be taken as a boundary condition for heavy particle jet flow.

(2) However, even when starting with a lower nozzle exit temperature, $4150 \mathrm{~K}$, the theoretical curves do not decline as fast as the experiments require. This leads to the suspicion that the jet is turbulent. The transition Reynolds number for an axisymmetric jet is about 2300. In our case, the torch orifice diameter $=6 \mathrm{~mm}$, plasma velocity

(a)

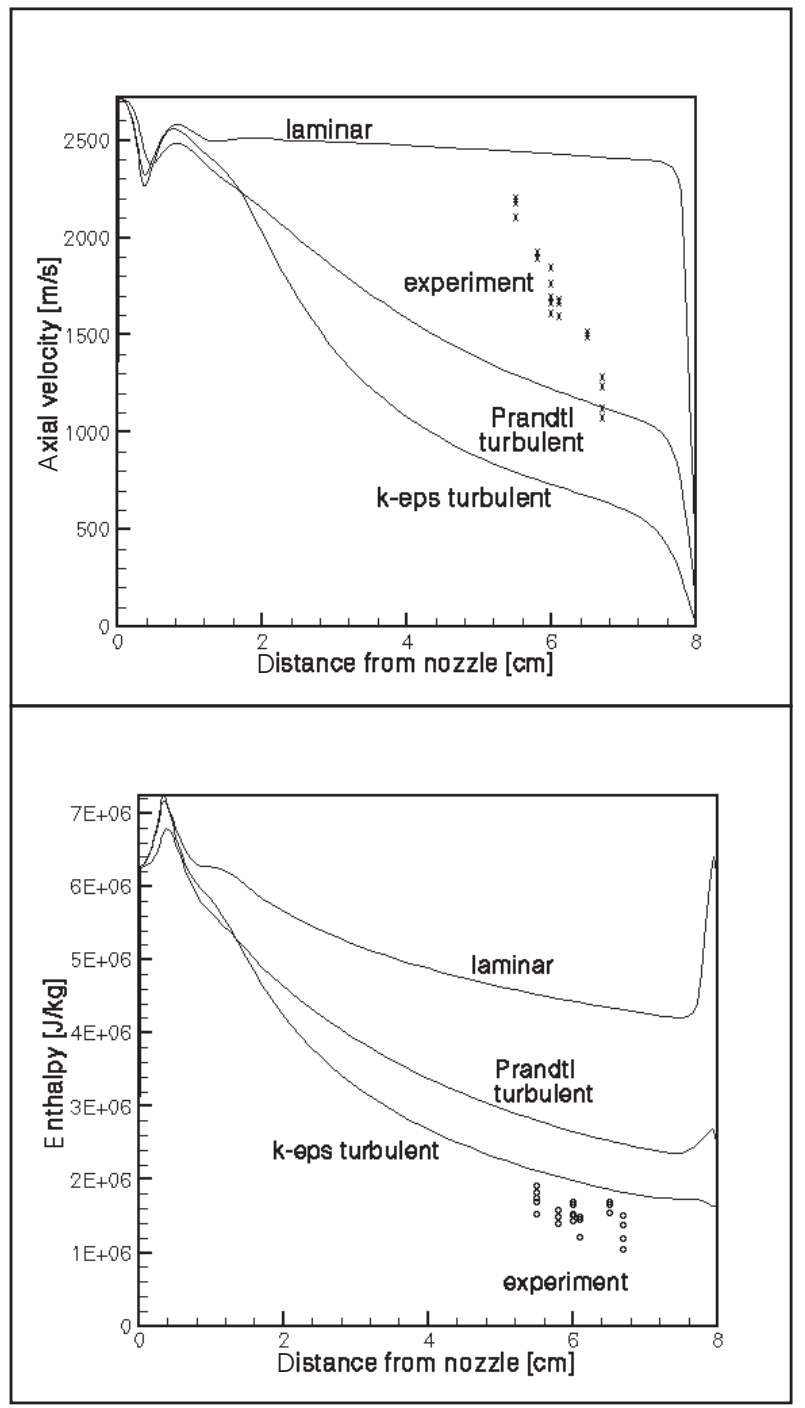

Fig. 2: (a) Axial jet velocity; (b) jet enthalpy - comparison of simulations and experiments 
$\sim 2700 \mathrm{~m} / \mathrm{s}$, density $\sim 0.1 \mathrm{~kg} / \mathrm{m}^{3}$, and viscosity $\sim 0.00016$ $\mathrm{kg} /(\mathrm{m} \cdot \mathrm{s})$, and thus the Reynolds number $\sim 10000$. This value decreases to some extent with increasing torch exit temperature, but always stays above the transition limit.

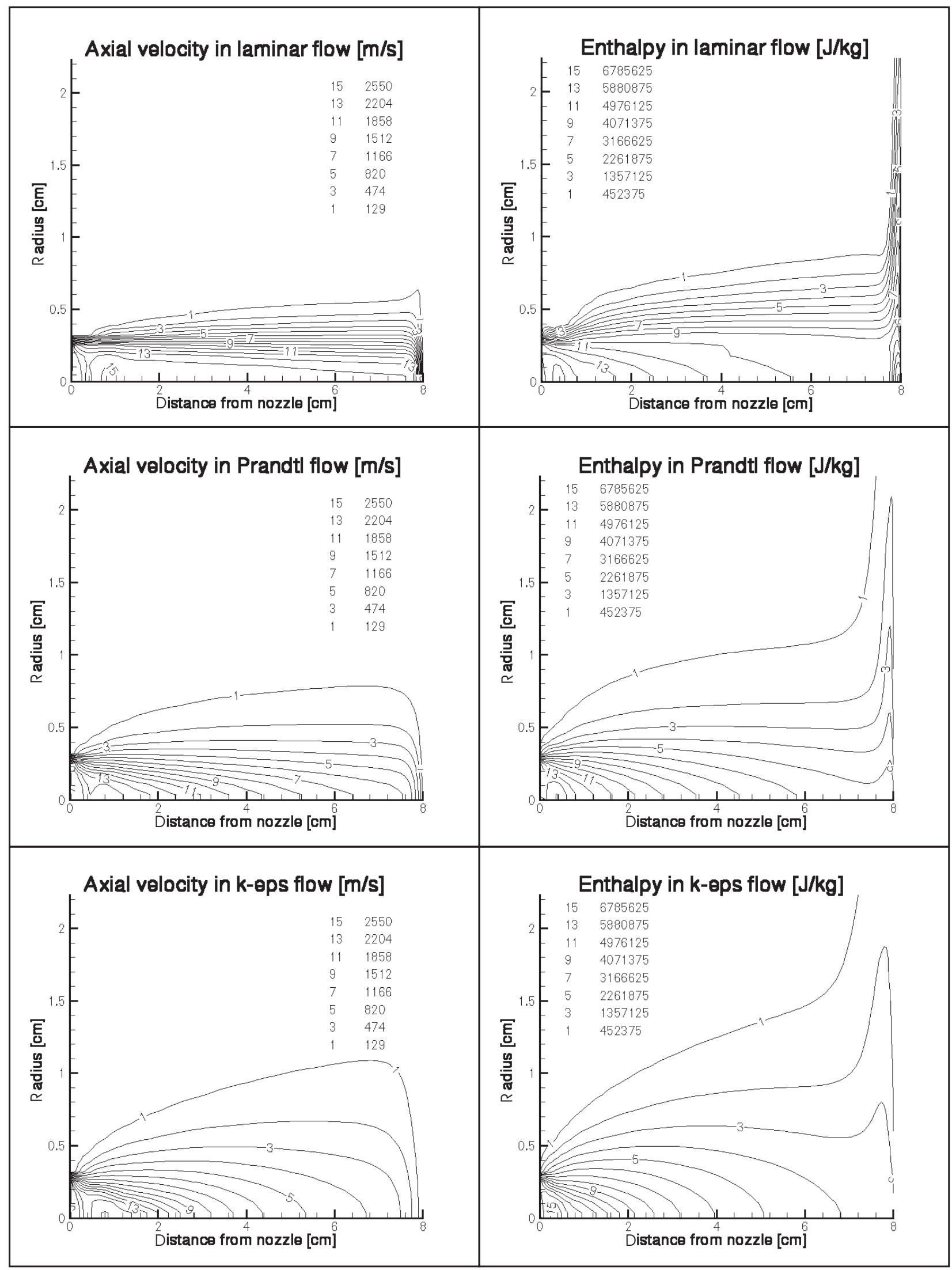

Fig. 3: Contours of the axial velocity and enthalpy for a laminar, Prandtl and k- $\varepsilon$ turbulent plasma jet 


\section{Turbulent flow modeling}

Modeling of the turbulence was approached in two ways:

(1) Using Prandtl's free shear layer model for eddy viscosity [5],

$$
\mu_{t}=C \cdot \rho \cdot \delta \cdot u_{\max }
$$

where $C$ is constant for the particular type of mixing layer, $C \sim 0.012$ for a round jet,

$\rho$ is local density,

$\delta$ is the shear layer thickness taken as the nozzle radius, and

$u_{\max }$ is the centerline jet velocity at a particular distance from the torch nozzle.

(2) Using the standard low Reynolds number k- $\varepsilon$ model of turbulence $[6,7]$.

Figure 2 shows that the character of the solution has changed drastically: One notes that the enthalpy corresponds quite well with the experimental measurements, both qualitatively and quantitatively. However, the agreement in the axial velocity is still rather poor. In the experimental data, which are unfortunately available only from a distance $55 \mathrm{~mm}$ away from the nozzle exit, the axial velocity falls from $\sim 2500 \mathrm{~m} / \mathrm{s}$ at $55 \mathrm{~mm}$ to $\sim 1200 \mathrm{~m} / \mathrm{s}$ at $67 \mathrm{~mm}$. In the simulation, the velocity falls sufficiently but much more gradually and over most of the distance to the nozzle.

The following remedies and explanations are available at the present time:

(1) One can modify the nozzle boundary conditions via an increase in the nozzle exit velocity and a decrease in the density, and pull the whole velocity profile up that way (either by decreasing the torch temperature further below $4000 \mathrm{~K}$, which is unrealistic, or by reducing the jet pressure further and thus causing greater overexpansion). However, this fix will not lead to a sudden drop in velocity, as the experiment suggests.

(2) The interpretation of the experimental data may be questioned on two counts: the assumption that the jet static presure equals the chamber pressure; and the finding that the pressure ratio in the entalpy probe measurements closer to the torch than $55 \mathrm{~mm}$ is so high that it does not allow velocity and enthalpy evaluation for those values. These points will need further clarification with the experimental researchers.

Finally, an illustrative comparison between individual computational cases is given in Figure 3. The two turbulent simulations produce much a shorter and much wider jet than the laminar case, as expected ( $k-\varepsilon$ leading to even more extensive entrainment of the ambient atmosphere than the Prandtl model). However, again, no turbulent modeling can reproduce the sudden velocity drop around $6 \mathrm{~cm}$ from the nozzle that the experiments suggest.

\section{Summary and conclusions}

This paper has reported the process of designing and analysing of a typical thermal plasma chemical vapor deposition system. It has shown the development of a theoretical model from a simple laminar model to a more demanding turbulent one, and has pointed out the reasons leading to such change in understanding. Also, close cooperation with the experimental researchers has been emphasized.

At the current stage, the benefits of the analysis undertaken are fourfold: (1) The simulation has confirmed the supersonic character of the flow; (2) Recirculation of the gas (incl. hydrocarbon precursor) in the region close to the torch exit has been brought to attention; (3) Numerous cross-checks and critical evaluations of the experiments were enforced, specific suggestions of further experiments have been made, some potentially erroneous assumptions in the experimental work have been exposed; and (4) Fruitful discussions have been initiated on various aspects of the deposition process, incl. the precursor flow pattern and its chemical composition, and boundary layer effects on chemistry.

Future work should include (1) a critical reevaluation of the enthalpy probe measurements, (2) better suited turbulence modeling, and (3) greater attention to the torch exit conditions. Only then trustworthy predictions and further optimization of the deposition process take place.

\section{Acknowledgement}

This project was in part carried out using computational resources provided under Research Grants AV ČR A1057001/120/00 and MSM/216200031.

\section{References}

[1] Jahn, E.: unpublished results, FG Plasma-Oberflächentechnik, Technische Universität Ilmenau, 1997

[2] Kolman, D., Heberlein, J., Pfender, E.: Influence of deposition parameters on diamond thermal plasma chemical vapor deposition with liquid feedstock injection. Diam. Rel. Mat. 7/1998, pp. 794-801

[3] Patankar, S. V.: Numerical Heat Transfer and Fluid Flow. Hemisphere Publishing, New York, 1980

[4] Schwenk, A., Sember V., Gravelle, D. V., Boulos, M. I., Nutsch, G.: Enthalpy probe measurements in supersonic plasma jets. VIII. Workshop Plasmatechnik, Technische Universität Ilmenau, Germany, Juni 2000

[5] Patankar, S. V.: Computation of Heat Transfer and Fluid Flow. Complementary notes, University of Minnesota

[6] Jones, W. P., Launder, B. E.: The prediction of laminarization with a two-equation model of turbulence. Int. J. Heat Mass Transfer 15, pp. 301-314

[7] Lam, C. K. G., Bremhorst, K.: A modified form of the $k-\varepsilon$ model for predicting wall turbulence. Transactions ASME 103, Sept. 1981, pp. 456-459.

Ing. David Kolman, PhD.

phone: +420224357541

e-mail:kolman@marian.fsik.cvut.cz

Department of Technical Mathematics Czech Technical University in Prague

Faculty of Mechanical Engineering

Karlovo nám. 13, 12135 Praha 2, Czech Republic 